\title{
Inelastic $H$ and $D$ atom scattering from Au(111) as benchmark for theory
}

Cite as: J. Chem. Phys. 150, 184704 (2019); https://doi.org/10.1063/1.5094693

Submitted: 05 March 2019 . Accepted: 24 April 2019 . Published Online: 14 May 2019

Hongyan Jiang (D), Yvonne Dorenkamp, Kerstin Krüger (D), and Oliver Bünermann (D)

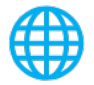

\section{ARTICLES YOU MAY BE INTERESTED IN}

Dynamics in reactions on metal surfaces: A theoretical perspective

The Journal of Chemical Physics 150, 180901 (2019); https://doi.org/10.1063/1.5096869

Stochastic wave packet approach to nonadiabatic scattering of diatomic molecules from metals

The Journal of Chemical Physics 150, 184105 (2019); https://doi.org/10.1063/1.5092698

Data sampling scheme for reproducing energies along reaction coordinates in highdimensional neural network potentials

The Journal of Chemical Physics 150, 134103 (2019); https://doi.org/10.1063/1.5078394

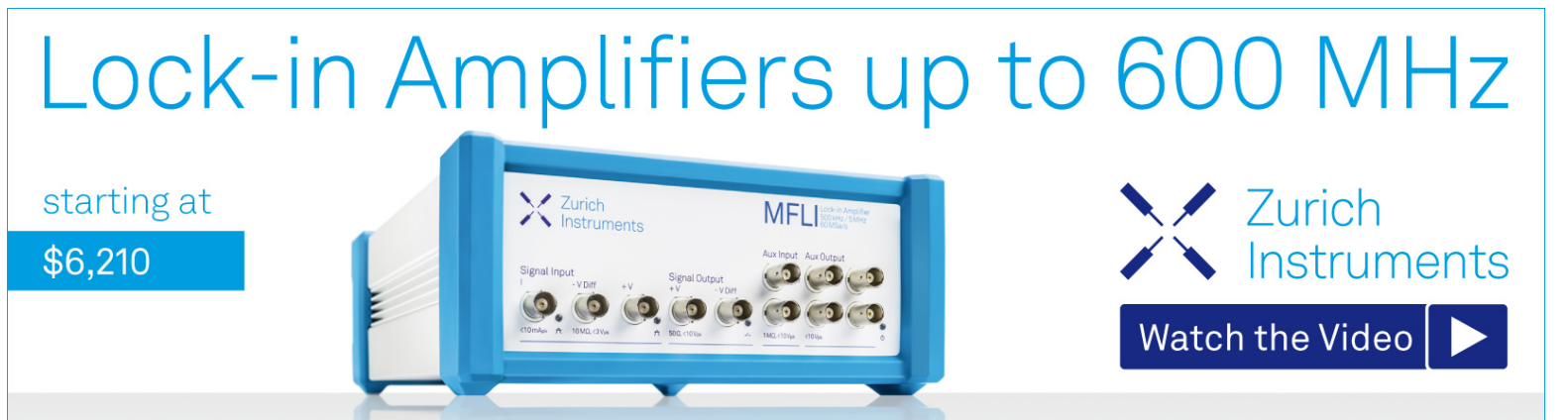




\title{
Inelastic $\mathrm{H}$ and $\mathrm{D}$ atom scattering from $\mathrm{Au}(\mathbf{1} 11)$ as benchmark for theory
}

\author{
Cite as: J. Chem. Phys. 150, 184704 (2019); doi: 10.1063/1.5094693 \\ Submitted: 5 March 2019 • Accepted: 24 April 2019 • \\ Published Online: 14 May 2019
}

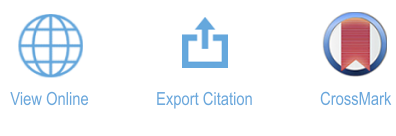

\section{Hongyan Jiang, ${ }^{1,2}$ (D) Yvonne Dorenkamp,' Kerstin Krüger, ${ }^{1}$ (D) and Oliver Bünermann ${ }^{1,2,3, a)}$ (D)}

\author{
AFFILIATIONS \\ ${ }^{1}$ Institute for Physical Chemistry, Georg-August University of Göttingen, Tammannstr. 6, 37077 Göttingen, Germany \\ ${ }^{2}$ Department of Dynamics at Surfaces, Max-Planck Institute for Biophysical Chemistry, Am Faßberg 11, \\ 37077 Göttingen, Germany \\ ${ }^{3}$ International Center for Advanced Studies of Energy Conversion, Georg-August University of Göttingen, \\ Tammannstr. 6, 37077 Göttingen, Germany
}

\begin{abstract}
a) Author to whom correspondence should be addressed: oliver.buenermann@chemie.uni-goettingen.de
\end{abstract}

\begin{abstract}
Efficient transfer of translational energy to electron-hole pair excitation involving multiple collisions dominates $\mathrm{H}$ atom collisions with metal surfaces. For this reason, $\mathrm{H}$ atom interaction with metal surfaces cannot be modeled within the commonly used Born-Oppenheimer approximation (BOA). This fact makes $\mathrm{H}$ atom scattering from metal surfaces an ideal model system for dynamics that go beyond the BOA. We chose the $\mathrm{H} / \mathrm{Au}(111)$ system as a model system to obtain a detailed dataset that can serve as a benchmark for theoretical models developed for describing electronically nonadiabatic processes at metal surfaces. Therefore, we investigate the influence of various experimental parameters on the energy loss in detail including isotopic variant, incidence translational energy, incidence polar and azimuthal angles, and outgoing scattering angles.
\end{abstract}

Published under license by AIP Publishing. https://doi.org/10.1063/1.5094693

\section{INTRODUCTION}

Hydrogen is the most prevalent element in the universe and understanding its surface dynamics has profound implications ranging from interstellar chemistry ${ }^{1}$ to maximizing performance of neutral beam injectors at the International Thermonuclear Experimental Reactor (ITER). ${ }^{2}$ Being one of the simplest model systems for energy conversion at surfaces, $\mathrm{H}$ atom scattering also provides a key to developing new theories for surface chemistry.

The high adsorption probability of atomic hydrogen on metal surfaces puzzled scientists for a long time. For an atom to adsorb to a surface, its translational energy has to be transferred to the solid. However, the $\mathrm{H}$ atom's low mass limits energy transfer to the lattice vibrations of the solid. In 1978, Norskov and Lundqvist suggested that energy transfer to electron-hole-pair excitations has to be considered to explain the high sticking probabilities. ${ }^{3}$ If electronic excitations play a role, the commonly used Born-Oppenheimer approximation (BOA) is not applicable and new theoretical models are required to model the surface dynamics of the hydrogen atom.
First evidence of electronic excitations in $\mathrm{H}$ atom interactions with surfaces was provided by experiments using Schottky diodes and metal-insulator-metal devices to measure the chemicurrent produced by $\mathrm{H}$ atom adsorption or by the $\mathrm{H}-\mathrm{H}$ recombination reaction on metal surfaces. ${ }^{4-7}$ The experimental findings inspired several theoretical studies. ${ }^{8-12}$ However, the only experimental observable here is the chemicurrent that is strongly dependent on the design of the sensor making comparison to theory difficult. Further theoretical studies focused on inelastic scattering ${ }^{13-15}$ and diffusion. ${ }^{16}$

Recently, we performed inelastic scattering experiments of $\mathrm{H}$ and $\mathrm{D}$ atoms from metal surfaces. ${ }^{17-19}$ A comparison between an insulator and a metal surface showed that a large amount of translational energy is transferred to electronic excitations of the metal surface in the scattering event, explaining the high adsorption probabilities. Kandratsenka et al. developed a theoretical model based on molecular dynamics (MD) simulations on a global full-dimensional potential energy surface (PES) to simulate the inelastic scattering experiments. ${ }^{20,21}$ Electronic excitations were included within the local-density electronic friction approximation 
(LDFA). ${ }^{22}$ The predictions of the model agreed well with observations from inelastic scattering experiments and provided an explanation of an apparent contradiction: a small isotope effect is seen in scattering, whereas a large isotope effect is seen in chemicurrent experiments.

So far, only a limited set of experimental parameters was available. In this paper, we present a large set of experimental data for one exemplary system $-\mathrm{H}$ atom scattering from $\mathrm{Au}(111)$. This gives a detailed picture of the scattering process and serves as a benchmark for newly developed theories describing the surface dynamics of $\mathrm{H}$ atoms on metals.

\section{METHODS}

A detailed description of the experimental setup is presented in Ref. 23. In short, a molecular beam of hydrogen halide molecules is formed in a supersonic expansion from a pulsed nozzle. After a skimmer, a UV laser beam intersects the molecular beam and dissociates the molecules. Part of the thereby formed $\mathrm{H}$ atoms passes a second skimmer and two apertures to form a well-collimated $\mathrm{H}$ atom beam with an incidence energy $E_{i n}$ and a narrow energy distribution. For high intensity beams, we use an $\mathrm{ArF}$ or $\mathrm{KrF}$ excimer laser for photolysis; for high resolution and tunability, we use a frequency doubled or tripled dye laser system.

The atom beam hits the sample surface held in ultrahigh vacuum in the main chamber. The sample is mounted on a six-axis manipulator that allows the transfer of the sample between the preparation and main chambers as well as the variation of the polar incidence angle $\vartheta_{\text {in }}$ and azimuthal incidence angle, $\varphi_{\text {in }}$ (with respect to the [10i] direction). The surface temperature, $T_{S}$, can be adjusted between 50 and $1500 \mathrm{~K}$, and heating is achieved by electron bombardment and cooling by a liquid nitrogen/helium flow cryostat. The preparation chamber houses an Ar ion canon for surface cleaning and an Auger electron spectrometer (AES) and low energy electron diffraction (LEED) for surface characterization. After the $\mathrm{H}$ atoms hit the surface, their final energy, $E_{f i n}$, and scattering angle, $\vartheta_{s}$, are measured by Rydberg atom tagging. ${ }^{24}$ Here, the $\mathrm{H}$ atoms are excited

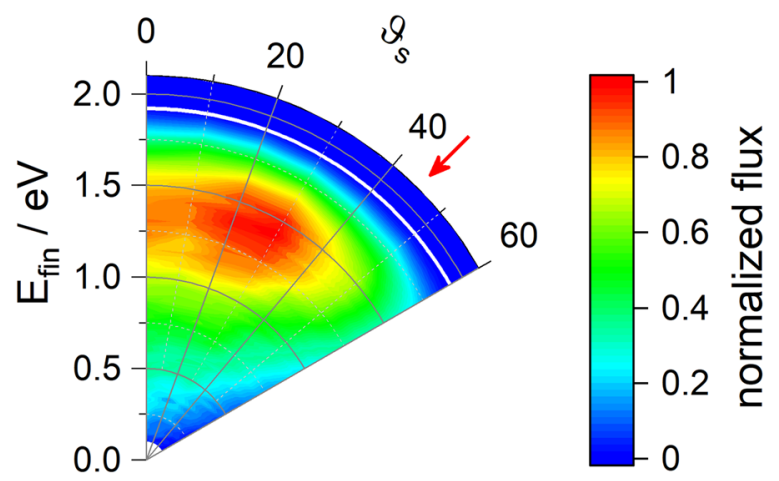

FIG. 1. Energy and angular resolved scattering distribution of $\mathrm{H}$ atoms scattered from a $A u(111)$ surface. We recorded energy loss spectra at various scattering angles in $10^{\circ}$ steps. The white line corresponds to the incidence energy of $E_{i n}=1.92 \mathrm{eV}$, and the red arrow marks the specular angle of $\vartheta_{i n}=45^{\circ}$. The distribution was obtained at $T_{S}=293 \mathrm{~K}$ and $\varphi_{\text {in }}=0^{\circ}$. to a long-lived Rydberg state by two photons. After passing a defined flight distance, they are field ionized and detected by a multichannel plate (MCP) detector. The detector is rotatable, allowing to record time-of-flight (TOF) distributions at a variety of scattering angles. The accessible angular range is limited by the incident beam on one side - the sum of incidence and scattering angle has to exceed $40^{\circ}-$ and by the size of the crystal on the other side-the line connecting the crystal edge and laser focus defines the largest scattering angle to be $75^{\circ}$. The time-of-flight spectra are converted to energy loss spectra applying the appropriate Jacobians.

Obtaining a fully energy and angular dispersed dataset is time consuming. Therefore, we do not measure the whole time-of-flight distributions at all scattering angles but only at a limited number of selected angles. Angular distributions are measured using a boxed car integrator to directly integrate over the TOF. In this way, we obtain a density distribution. Using the TOF distributions measured for selected angles, we calculate the corresponding
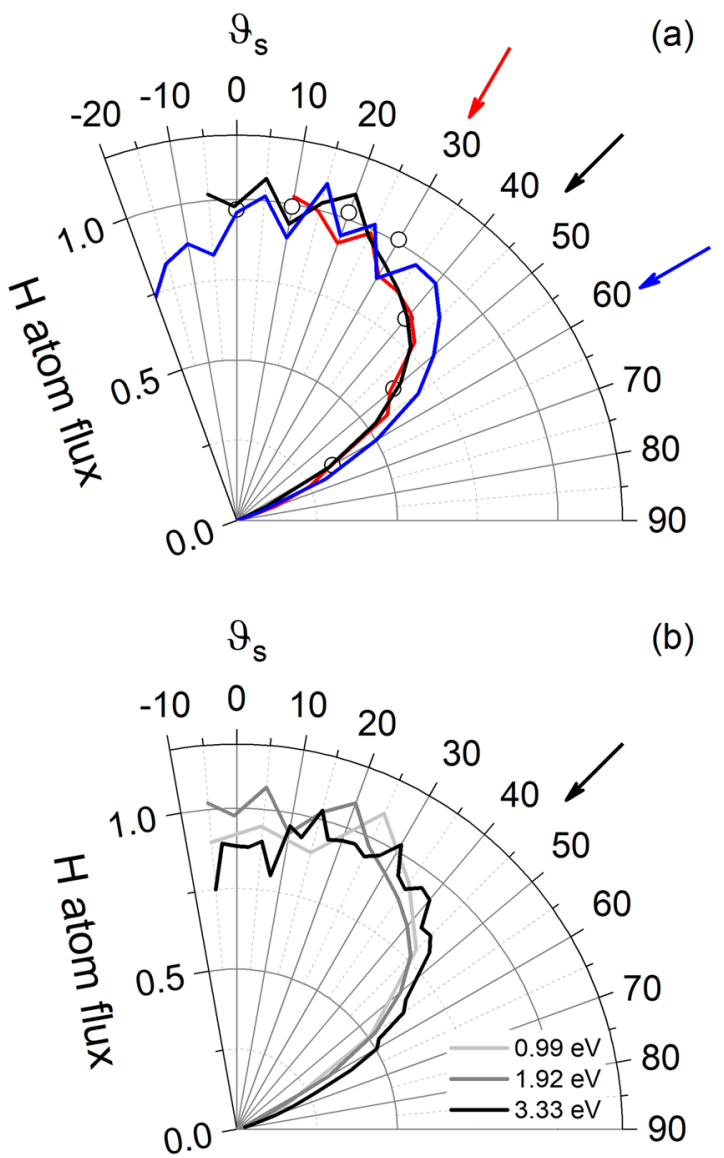

FIG. 2. Angular distributions for $H$ atoms scattered from $A u(111)$. Panel (a) shows results for one incidence energy $(1.92 \mathrm{eV})$ and three polar incidence angles. The arrows mark the corresponding specular angles. The open symbols show the angular distribution for $\vartheta_{i}=45^{\circ}$ obtained from the data shown in Fig. 1. Panel (b) shows angular distributions for three different incidence energies and one polar incidence angle. The black arrow marks the specular angle of $45^{\circ}$. All distributions were obtained at $T_{S}=293 \mathrm{~K}$ and $\varphi_{\text {in }}=0^{\circ}$. 
average velocities of the scattered $\mathrm{H}$ atoms. The average velocity of the scattered $\mathrm{H}$ atoms roughly scales linear with the scattering angle. Using the obtained linear function for the average velocity in dependence of scattering angle, we perform an approximate density to flux conversion of the angular distributions.

\section{RESULTS}

Figure 1 shows a representative angle-resolved translational energy distribution of $\mathrm{H}$ atoms scattered from a $\mathrm{Au}(111)$ surface. The white line at $1.92 \mathrm{eV}$ marks the incidence translational energy and the red arrow at $45^{\circ}$ indicates the specular angle. The $\mathrm{H}$ beam is incident along the [10ī] direction and the surface is at $T_{S}=293 \mathrm{~K}$. Due to experimental limitations, we cannot measure the complete distribution. The scattering distribution consists of one broad feature peaking at $\vartheta_{s} \sim 25^{\circ}$ and $E_{\text {fin }} \sim 1.4 \mathrm{eV}$, resulting in a most probable energy loss of $\sim 0.5 \mathrm{eV}$. The average energy loss, $E_{i n}-E_{\text {fin }}$, is estimated to $\sim 0.75 \mathrm{eV}$, which corresponds to $\sim 39 \%$ of the incidence energy. The broad angular distribution peaking between the specular angle and surface normal suggests that, on average, the $\mathrm{H}$ atoms undergo several collisions with the surface in the scattering event. The large energy loss is due to an efficient energy transfer to electron-hole-pair excitations. ${ }^{17-19}$

In the following, three aspects of the scattering experiments are discussed in detail: First, the influence of the incidence parameters of the $\mathrm{H}$ atom beam, second the influence of the azimuthal orientation of the crystal, and third the isotope effect.

\section{Incident beam conditions}

The incident beam conditions include the incidence energy $E_{i n}$ and the polar incidence angle $\vartheta_{\text {in }}$. In this work, we covered an incidence energy range of $\sim 0.9-3.3 \mathrm{eV}$. Going to lower incidence energies is, in principle, possible, but the large sticking probabilities for $\mathrm{H}$ on $\mathrm{Au}(111)$ for incidence energies $<0.9 \mathrm{eV}$ prohibit scattering experiments. ${ }^{19}$ The size of the crystal and the geometry of the sample mount limit the polar incidence angle to a maximum of $75^{\circ}$. Instead of measuring the complete angle-resolved translational energy distribution, we measured energy integrated angular distributions and energy loss spectra at selected scattering angles, making direct comparisons between results for different incidence conditions straight forward.

Integrating the distribution in Fig. 1 over energy, we obtain the angular distribution. The corresponding points are shown as open symbols in panel (a) of Fig. 2. The solid lines show angular distributions for three polar incidence angles and otherwise identical experimental conditions obtained by directly integrating over the TOF and applying the approximate density to flux conversion described above. The angular distributions for $\vartheta_{\text {in }}=30^{\circ}$ and $45^{\circ}$ are identical, the one for $\vartheta_{\text {in }}=60^{\circ}$ shows slightly increased intensity at large scattering angles. All three are broad and show no structure. Panel (b) of Fig. 2 shows the dependency of the angular distributions on the incidence energy for one incidence angle. We observe no dependency of the angular distribution on the incidence energy within the experimental error.

We are not able to cover the full angular range in our experiment. That is why we cannot derive angular integrated energy loss spectra. Instead, we compare energy loss spectra at specific scattering angles, which correspond to slices of Fig. 1. Figure 3 shows the dependency of the energy loss spectra on the polar incidence and scattering angles for two incidence energies. For a fixed incidence angle of $45^{\circ}$ [Figs. 3(a) and 3(c)], the energy loss increases for decreasing scattering angle; $\mathrm{H}$ atoms leaving the surface along the surface normal lost more energy than the atoms leaving at the

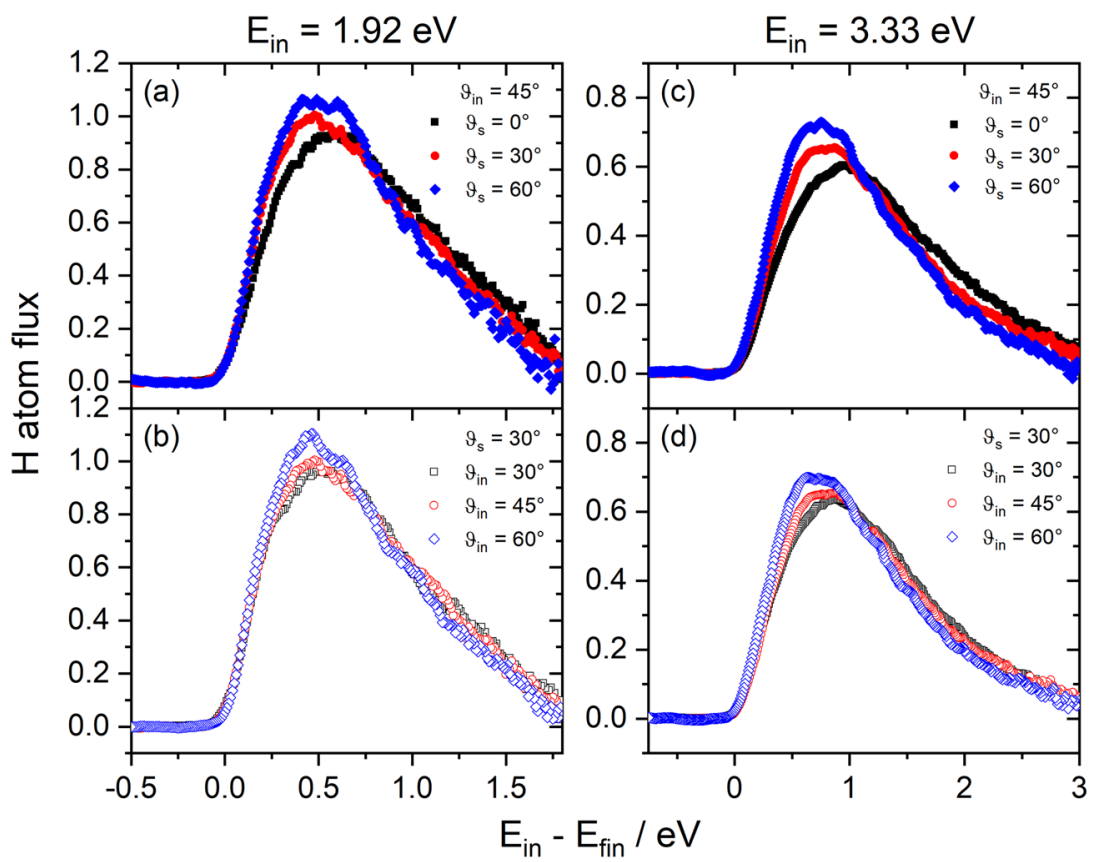

FIG. 3. Dependence of the energy loss spectrum of $\mathrm{H}$ atoms scattered form $\mathrm{Au}(111)$ on the polar incidence and scattering angles. Panels (a) and (b) show results for an incidence energy of $1.92 \mathrm{eV}$ and panels (c) and (d) for $3.33 \mathrm{eV}$. Panels (a) and (c) show the dependence on the polar scattering angle for one incidence angle, and panels (b) and (d) show the dependence on the polar incidence angle for one scattering angle. All spectra were obtained at $T_{S}=293 \mathrm{~K}$ and $\varphi_{\text {in }}=0^{\circ}$. 


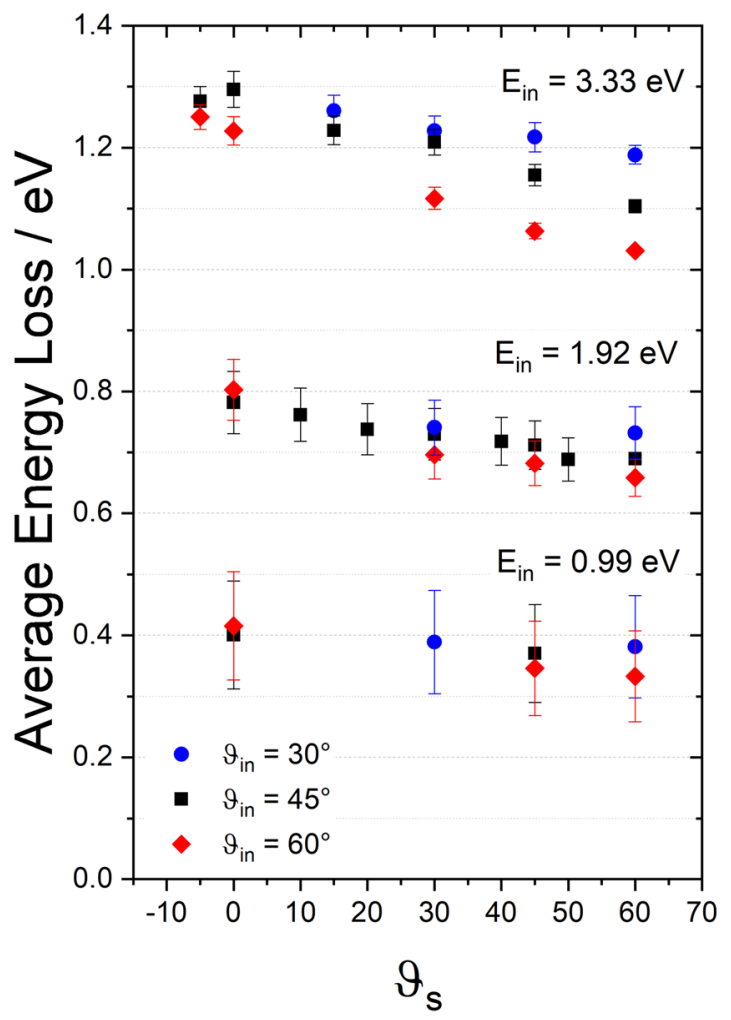

FIG. 4. Average energy loss of $\mathrm{H}$ atoms scattered from $\mathrm{Au}(111)$ in dependence of polar incidence angle, scattering angle, and incidence energy. All results were obtained at $T_{S}=293 \mathrm{~K}$ and $\varphi_{\text {in }}=0^{\circ}$.

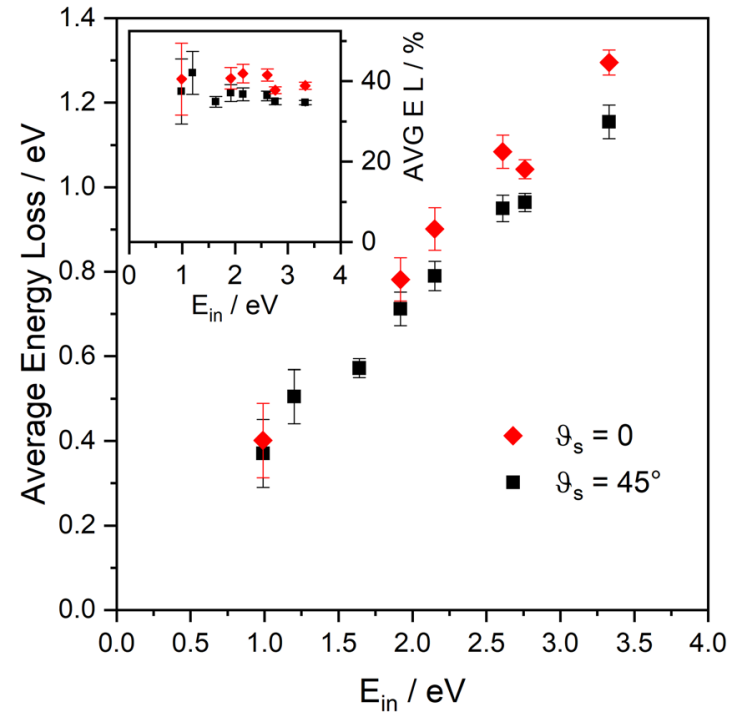

FIG. 5. Dependence of average energy loss of $\mathrm{H}$ atoms scattered from $\mathrm{Au}(111)$ on incidence energy for one incidence angle and two scattering angles. The inset shows the average energy loss scaled by incidence energy. All results were obtained at $T_{S}=293 \mathrm{~K}$ and $\varphi_{\text {in }}=0^{\circ}$.

specular angle. The shape of the spectrum remains the same, showing one broad feature. Panels (b) and (d) of Fig. 3 show example spectra for one scattering angle of $30^{\circ}$ and three different incidence angles. Here, a similar trend is visible: the closer the incidence angle is to the surface normal, the more energy is lost in the scattering event.
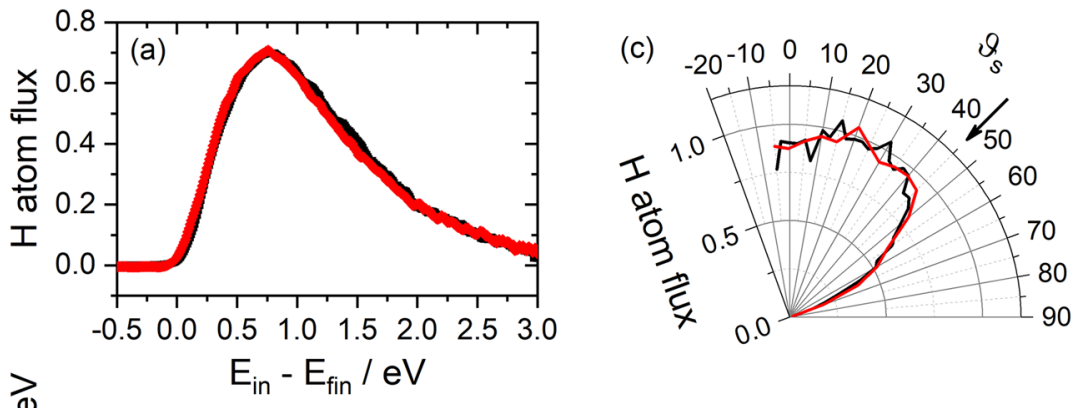

FIG. 6. Influence of crystal azimuthal orientation: Panel (a) shows the energy loss spectra of $\mathrm{H}$ atoms scattered from $\mathrm{Au}(111)$ for $E_{\text {in }}=3.33 \mathrm{eV}$ and $\vartheta_{\text {in }}=45^{\circ}$ for two azimuthal orientations, $\varphi_{\text {in }}=0^{\circ}$ $([10 \overline{1}]$, black $)$ and $\varphi_{\text {in }}=30^{\circ}([11 \overline{2}]$, red). Panel (b) shows the average energy loss in dependence of the scattering angle for both surface directions. The points marked with the gray circle correspond to the spectra shown in panel (a). Panel (c) shows angular distributions for $E_{\text {in }}=3.33 \mathrm{eV}$ and $\vartheta_{\text {in }}=45^{\circ}$ for both directions-the arrow marks the specular angle. Panel (d) shows the $\mathrm{H}$ atom flux in dependence of the crystal azimuthal orientation for $E_{\text {in }}=3.33 \mathrm{eV}, \vartheta_{\text {in }}=60^{\circ}$, and $\vartheta_{s}=0^{\circ}$. All results were obtained at $T_{S}=293 \mathrm{~K}$.

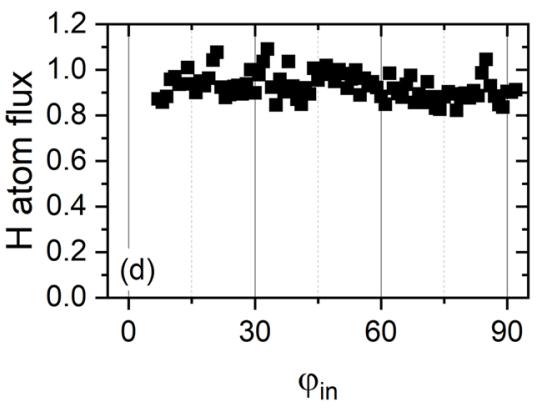


Because the energy loss spectra show only a single feature and have similar shape, they can all be characterized by their average energy loss. Figure 4 shows the average energy loss as a function of the scattering angle for three incidence energies and three polar incidence angles. Here, the above-mentioned trends are clearly visible. The energy loss decreases with increasing incidence and scattering angles. The higher the incidence energy, the stronger the dependencies become. For a scattering angle of $0^{\circ}$, the energy loss becomes independent of the incidence angle. The reason for larger error bars for lower incidence energies is the sticking probability of $\mathrm{H}$ to $\mathrm{Au}(111)$ : It increases from $48 \%$ for an incidence energy of $3.33 \mathrm{eV}$ to $79 \%$ for $0.99 \mathrm{eV},{ }^{19}$ leading to a lower scattering signal for smaller incidence energies.

Figure 5 shows the dependence of the average energy loss on the incidence energy for one incidence angle and two scattering angles. In the studied energy range, the energy loss scales nearly linearly with incidence energy. The inset shows the average energy loss scaled to the incidence energy. The $\mathrm{H}$ atoms lose $~ 35 \%-40 \%$ of their energy in the scattering event. A small deviation from linear scaling is observed; the percentage energy loss increases with decreasing incidence energy. Furthermore, $\mathrm{H}$ atoms observed at $\vartheta_{s}$ $=0^{\circ}$ transferred more translational energy to the surface than the atoms observed at $\vartheta_{s}=45^{\circ}$.

\section{Crystal azimuthal orientation}

We studied the effect of the azimuthal orientation of the crystal on the scattering event in detail. For this study, we chose an incidence energy of $3.33 \mathrm{eV}$. Figure 6 summarizes the obtained data. Figure 6(a) shows a representative comparison of two energy loss spectra recorded for two different azimuthal orientations with otherwise identical experiential conditions. Both spectra are indistinguishable. Also, the angular distributions-shown in panel (c) - are identical for both crystal orientations. We made comparisons like the one shown in Fig. 6(a) for many different scattering angles. Figure 6(b) shows the obtained average energy losses. We do not observe a dependency on the azimuthal orientation within our experimental precision. Figure 6 (d) shows the $\mathrm{H}$ atom flux in dependence of the crystal azimuthal orientation for a scattering geometry of $\vartheta_{\text {in }}=60^{\circ}$ and $\vartheta_{s}=0^{\circ}$. The flux is independent of the crystal orientation.

\section{The isotope effect}

A detailed study of the isotope effect is shown in Fig. 7. Figure 7(a) compares the angular distributions at identical experimental conditions for $\mathrm{H}$ and $\mathrm{D}$ atoms scattered from a $\mathrm{Au}(111)$ surface. No difference is observable. Figure 7 (b) shows one representative comparison of energy loss spectra. Both spectra are very similar, differences are observed for low energy loss, where D atoms show more intensity than $\mathrm{H}$ atoms. For large energy losses, the spectra are nearly identical. Figure 7 (c) shows the average energy loss and the isotope effect in dependence of incidence energy for two scattering angles. The average energy loss for $\mathrm{H}$ and $\mathrm{D}$ is similar in all cases resulting in a small isotope effect of $\Delta E_{H} / \Delta E_{D} \sim 1.1$. The isotope effect is independent of the incidence angle and incidence energy.
Theoretical simulations allow a separation of the phonon and electronic part of the energy dissipation to the surface. According to Ref. 18, most of the dissipated energy goes into electronhole-pair excitations ( $90 \%$ for $\mathrm{H}, 79 \%$ for D). To experimentally

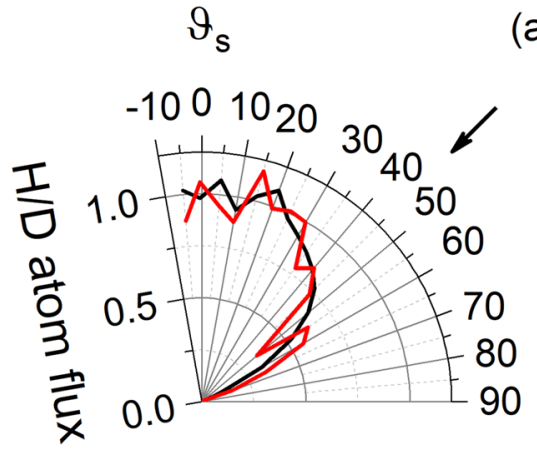

(a)
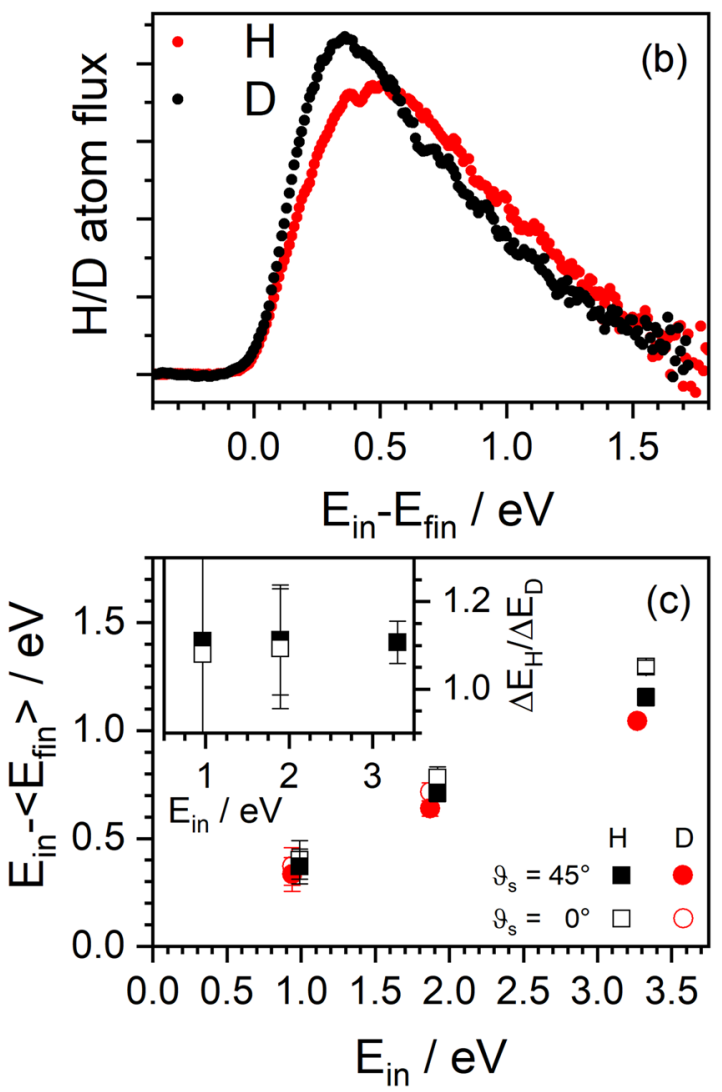

FIG. 7. The isotope effect: Panel (a) shows the angular distribution for $1.9 \mathrm{eV} \mathrm{H}$ and $\mathrm{D}$ atoms scattered from $\mathrm{Au}(111)$. The black arrow marks the specular angle. Panel (b) shows one representative comparison of the energy loss spectra of $\mathrm{H}$ and $\mathrm{D}$ for the experimental parameters $E_{H, \text { in }}=1.92 \mathrm{eV}, E_{D, \text { in }}=1.87 \mathrm{eV}, \vartheta_{\text {in }}=\vartheta_{s}=45^{\circ}$, and $\varphi_{\text {in }}=0^{\circ}$. Panel (c) shows the average energy loss and the isotope effect for three different incidence energies and two scattering angles for $\vartheta_{\text {in }}=45^{\circ}, \varphi_{\text {in }}=0^{\circ}$. All data were obtained at $T_{S}=293 \mathrm{~K}$. 


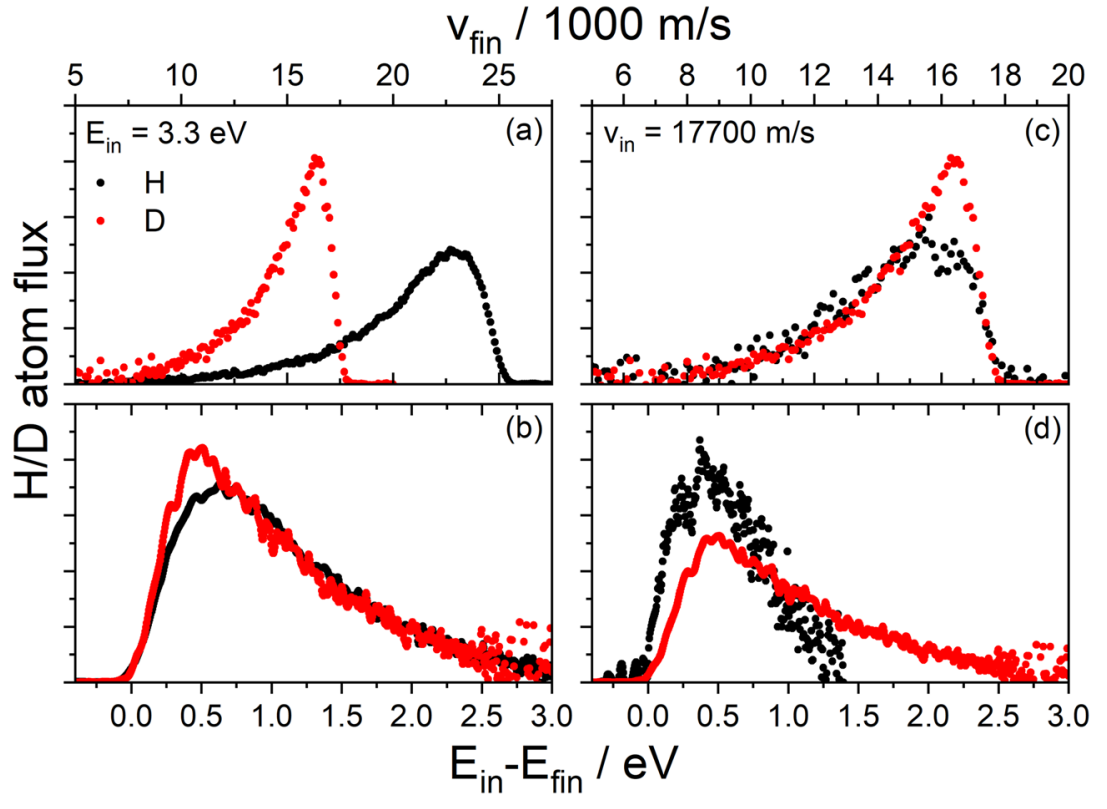

FIG. 8. $H$ and $D$ atom scattering from $\mathrm{Au}(111)$. (a) and (b) show the speed and energy loss distributions for identical incidence energies for $H$ and $D$. The experimental conditions are $E_{H, \text { in }}=3.33 \mathrm{eV}$, $E_{D \text { in }}=3.27 \mathrm{eV}, \vartheta_{\text {in }}=\vartheta_{s}=45^{\circ}, \varphi_{\text {in }}=0^{\circ}$, and $T_{S}=293 \mathrm{~K}$. (c) and (d) show the speed and energy loss distributions for equal incidence speeds $(=17700 \mathrm{~m} / \mathrm{s})$ of $H$ and D. Black corresponds to $H$ and red to $\mathrm{D}$. disentangle the phonon and electronic contributions, measurements at equal velocity were performed. Figure 8 shows a set of measurements comparing the energy loss for $\mathrm{H}$ and $\mathrm{D}$ atoms first having the same incidence energy and second the same incidence velocity. Thereby, the $\mathrm{D}$ atom beam is kept the same whereas the kinetic energy of the $\mathrm{H}$ atom beam is varied. Figures $8(\mathrm{a})$ and $8(\mathrm{c})$ show the corresponding velocity distributions while panels (b) and (d) the energy loss spectra, panels (a) and (b) compare spectra for equal incidence energy, and panels (c) and (d) for equal incidence velocities.

Assuming that the electronic coupling scales with velocity only, it should be the same for $\mathrm{H}$ and $\mathrm{D}$ when both have equal velocities. The difference between the observed energy losses is then caused by the difference in phonon coupling due to different masses. For $v_{H}=v_{D}, \mathrm{D}$ atoms have twice the energy $2 \cdot E_{H}=E_{D}$. Assuming phonon excitation scales linearly with mass, $D$ atoms lose $2 \times 2$ times more energy to phonon excitation, $\Delta E_{D, p}=4 \cdot \Delta E_{H, p}$, but the loss to electronic excitation should be equal for $\mathrm{H}$ and $\mathrm{D}: \Delta E_{D, e}=\Delta E_{H, e}$. The overall loss is the sum of both components. By solving the equations for $\Delta E_{H, e}$ and $\Delta E_{D, e}$, we can disentangle the phonon and the electronic contribution to the energy loss. Instead of using the average energy loss, the most probable energy loss is used in this case. It is not possible to disentangle both contributions for multibounce collisions, meaning we have to focus on single bounce events for this analysis. According to the theoretical model, the most probable energy loss approximately represents the average energy loss for single bounce events. ${ }^{18}$ In the above example, the most probable energy losses are $380 \mathrm{meV}$ for $\mathrm{H}$ and $500 \mathrm{meV}$ for $\mathrm{D}$, which results in $\Delta E_{D, e}=\Delta E_{H, e}=340 \mathrm{meV}$ and $\Delta E_{H, p}=1 / 4 \cdot \Delta E_{D, p}=40 \mathrm{meV}$. Therefore, this simple analysis yields that $89 \%$ of the transferred energy goes to electronic excitations in the case of $\mathrm{H}$ and $68 \%$ in the case of $\mathrm{D}$, close to the values predicted by the theoretical simulation.

\section{DISCUSSION}

The energy loss spectra as well as the angular distributions of $\mathrm{H}$ atoms scattered from $\mathrm{Au}(111)$ surfaces show small dependencies on the investigated experimental conditions. The $\mathrm{H}$ atoms lose on average $35 \%-40 \%$ of their incidence energy. The angular distributions are broad and structureless, and a weak dependency on the incidence angle is observable. The broad angular distributions indicate no direct scattering event. Still, there is clearly no trappingdesorption process, since the energy distributions of the scattered $\mathrm{H}$ atoms are not thermal. The $\mathrm{H}$ atoms experience a limited number of bounces before leaving the surface again. Theoretical calculations predict an average number of 2-3 depending on the incidence energy. ${ }^{19} \mathrm{H}$ atoms that underwent a single collision are preferentially observed close to the specular angle, while $\mathrm{H}$ atoms that underwent several collisions are expected to follow a $\cos \theta$ angular distribution peaking at the surface normal. This explains the observed scattering angle dependency of the energy loss: At specular angle, single bounce events are observed with higher probability, while at surface normal, multibounce events dominate. As the energy loss increases with the number of collisions, ${ }^{18}$ the average energy loss increases with decreasing scattering angle.

The energy loss also shows a weak dependency on the incidence angle: The closer the incidence angle is to the surface normal, the more energy is lost. One possible explanation is a small normal energy scaling of the electronic part of the energy loss: the larger the normal energy, the deeper the $\mathrm{H}$ atoms can penetrate the potential and experience a higher electron density, leading to an increased energy loss to electron-hole-pair excitations. Assuming that the phonon part of the energy loss can approximately be described within the hard cube model, ${ }^{25}$ it should scale with $\cos ^{2} \vartheta_{i n}$, which is also in accordance with the experimental findings. 
We did not observe any effect of the azimuthal crystal orientation on the experimental results. In this context, one should note that the $\mathrm{Au}(111)$ surface reconstructs in the so-called herringbone reconstruction and is not the optimal model system for studying the influence of the azimuthal orientation. ${ }^{26}$ The surface reconstruction could mask an azimuthal dependency due to the random orientation of its domains. A nonreconstructing surface like $\mathrm{Ag}(111)$ would be a more ideal candidate for such experiments. Still, we can exclude a strong influence of the crystal orientation.

Replacing $\mathrm{H}$ with its heavier isotope $\mathrm{D}$ leads to small changes. The angular distributions are identical. $\mathrm{H}$ atoms, on average, lose more energy than $\mathrm{D}$ atoms giving an isotope effect of $\Delta E_{H} / \Delta E_{D}$ $\sim$ 1.1. The isotope effect is independent of the incidence energy and angle. The small isotope effect is explained by a compensation of two mass dependent effects: ${ }^{18}$ The energy transfer to lattice vibrations scales with $m$ and, therefore, is larger for D than for $\mathrm{H}$. The energy transfer to electron-hole-pair excitations scales with the velocity of the atoms, thus with $1 / \sqrt{m}$, and is smaller for $\mathrm{D}$ than for H. Here, both effects nearly compensate each other, resulting in a small isotope effect. Measuring the isotope effect at equal velocities enabled us to estimate the amount of energy transferred to lattice vibrations and to electron-hole-pair excitations. The electronic part dominates the energy loss with $89 \%$ for $\mathrm{H}$ and $69 \%$ for $\mathrm{D}$. The obtained ratios are in good agreement with theoretical predictions.

\section{CONCLUSION}

In this paper, we presented a detailed dataset for $\mathrm{H}$ and $\mathrm{D}$ atom scattering from the $\mathrm{Au}(111)$ surface for the energy range of 0.94-3.33 eV that provides a valuable benchmark for theoretical models of surface chemistry going beyond the BOA. The angular resolved energy loss distributions consist of a single broad feature. The average energy loss shows weak dependencies on the experimental conditions. The azimuthal orientation of the crystal has no observable effect on the scattering results. The compensation of two mass dependent effects leads to a small isotope effect.

\section{ACKNOWLEDGMENTS}

O.B. is thankful to A. M. Wodtke for continuous support in every aspect of the work. We thank D. J. Auerbach for fruitful discussions, X. Yang and C. Xiao for helping us setting up RAT, and R. Bürsing for helping in designing the experimental apparatus. We acknowledge support from the SFB1073 under Project A04, the Deutsche Forschungsgemeinschaft (DFG), the Ministerium für Wissenschaft und Kultur (MWK) Niedersachsen, and the Volkswagenstiftung under Grant No. INST 186/902-1.

\section{REFERENCES}

${ }^{1}$ D. Hollenbach and E. E. Salpeter, "Surface recombination of hydrogen molecules," Astrophys. J. 163, 155 (1971).

${ }^{2}$ G. Federici, C. H. Skinner, J. N. Brooks, J. P. Coad, C. Grisolia, A. A. Haasz, A. Hassanein, V. Philipps, C. S. Pitcher, J. Roth, W. R. Wampler, and D. G. Whyte, "Plasma-material interactions in current tokamaks and their implications for next step fusion reactors," Nucl. Fusion 41, 1967-2137 (2001).
${ }^{3}$ J. K. Norskov and B. I. Lundqvist, "Correlation between sticking probability and adsorbate-induced electron-structure,” Surf. Sci. 89, 251-261 (1979).

${ }^{4}$ H. Nienhaus, H. S. Bergh, B. Gergen, A. Majumdar, W. H. Weinberg, and E. W. McFarland, "Electron-hole pair creation at Ag and Cu surfaces by adsorption of atomic hydrogen and deuterium," Phys. Rev. Lett. 82, 446-449 (1999).

${ }^{\mathbf{5}}$ B. Gergen, H. Nienhaus, W. H. Weinberg, and E. W. McFarland, "Chemically induced electronic excitations at metal surfaces," Science 294, 2521-2523 (2001).

${ }^{6}$ B. Mildner, E. Hasselbrink, and D. Diesing, "Electronic excitations induced by surface reactions of $\mathrm{H}$ and $\mathrm{D}$ on gold," Chem. Phys. Lett. 432, 133-138 (2006).

${ }^{7}$ B. Schindler, D. Diesing, and E. Hasselbrink, "Electronic excitations in the course of the reaction of $\mathrm{H}$ with coinage and noble metal surfaces: A comparison," Z. Phys. Chem. 227, 1381-1395 (2013).

${ }^{8}$ J. R. Trail, M. C. Graham, D. M. Bird, M. Persson, and S. Holloway, "Energy loss of atoms at metal surfaces due to electron-hole pair excitations: First-principles theory of 'chemicurrents,"' Phys. Rev. Lett. 88, 166802 (2002).

${ }^{9}$ J. R. Trail, D. M. Bird, M. Persson, and S. Holloway, "Electron-hole pair creation by atoms incident on a metal surface," J. Chem. Phys. 119, 4539-4549 (2003).

${ }^{10}$ M. S. Mizielinski, D. M. Bird, M. Persson, and S. Holloway, "NewnsAnderson model of chemicurrents in H/Cu and H/Ag," Surf. Sci. 602, 2617-2622 (2008).

${ }^{11}$ D. M. Bird, M. S. Mizielinski, M. Lindenblatt, and E. Pehlke, "Electronic excitation in atomic adsorption on metals: A comparison of $a b$ initio and model calculations,” Surf. Sci. 602, 1212-1216 (2008).

${ }^{12}$ M. S. Mizielinski and D. M. Bird, "Accuracy of perturbation theory for nonadiabatic effects in adsorbate-surface dynamics," J. Chem. Phys. 132, 184704 (2010).

${ }^{13}$ M. Pavanello, D. J. Auerbach, A. M. Wodtke, M. Blanco-Rey, M. Alducin, and G. J. Kroes, "Adiabatic energy loss in hyperthermal $\mathrm{H}$ atom collisions with $\mathrm{Cu}$ and Au: A basis for testing the importance of nonadiabatic energy loss," J. Phys. Chem. Lett. 4, 3735-3740 (2013).

${ }^{14}$ S. M. Janke, M. Pavanello, G. J. Kroes, D. Auerbach, A. M. Wodtke, and A. Kandratsenka, "Toward detection of electron-hole pair excitation in $\mathrm{H}$-atom collisions with $\mathrm{Au}(111)$ : Adiabatic molecular dynamics with a semi-empirical full-dimensional potential energy surface," Z. Phys. Chem. 227, 1467-1490 (2013).

${ }^{15}$ G. J. Kroes, M. Pavanello, M. Blanco-Rey, M. Alducin, and D. J. Auerbach, "Ab initio molecular dynamics calculations on scattering of hyperthermal $\mathrm{H}$ atoms from $\mathrm{Cu}(111)$ and $\mathrm{Au}(111)$, , J. Chem. Phys. 141, 054705 (2014).

${ }^{16}$ M. Blanco-Rey, J. I. Juaristi, R. D. Muino, H. F. Busnengo, G. J. Kroes, and M. Alducin, "Electronic friction dominates hydrogen hot-atom relaxation on Pd(100)," Phys. Rev. Lett. 112, 103203 (2014).

${ }^{17}$ O. Buenermann, H. Y. Jiang, Y. Dorenkamp, A. Kandratsenka, S. M. Janke, D. J. Auerbach, and A. M. Wodtke, "Electron-hole pair excitation determines the mechanism of hydrogen atom adsorption," Science 350, 1346-1349 (2015).

${ }^{18}$ A. Kandratsenka, H. Y. Jiang, Y. Dorenkamp, S. M. Janke, M. Kammler, A. M. Wodtke, and O. Buenermann, "Unified description of H-atom-induced chemicurrents and inelastic scattering," Proc. Natl. Acad. Sci. U. S. A. 115, 680-684 (2018).

${ }^{19}$ Y. Dorenkamp, H. Y. Jiang, H. Kockert, N. Hertl, M. Kammler, S. M. Janke, A. Kandratsenka, A. M. Wodtke, and O. Buenermann, "Hydrogen collisions with transition metal surfaces: Universal electronically nonadiabatic adsorption," J. Chem. Phys. 148, 034706 (2018).

${ }^{20}$ S. M. Janke, D. J. Auerbach, A. M. Wodtke, and A. Kandratsenka, "An accurate full-dimensional potential energy surface for $\mathrm{H}-\mathrm{Au}(111)$ : Importance of nonadiabatic electronic excitation in energy transfer and adsorption," J. Chem. Phys. 143, 124708 (2015).

${ }^{21}$ M. Kammler, S. M. Janke, A. Kandratsenka, and A. M. Wodtke, "Genetic algorithm approach to global optimization of the full-dimensional potential energy surface for hydrogen atom at fcc-metal surfaces," Chem. Phys. Lett. 683, 286-290 (2017).

${ }^{22}$ Y. G. Li and G. Wahnstrom, "Molecular-dynamics simulation of hydrogen diffusion in palladium,” Phys. Rev. B 46, 14528-14542 (1992). 
${ }^{23}$ O. Buenermann, H. Y. Jiang, Y. Dorenkamp, D. J. Auerbach, and A. M. Wodtke, "An ultrahigh vacuum apparatus for $\mathrm{H}$ atom scattering from surfaces," Rev. Sci. Instrum. 89, 094101 (2018).

${ }^{24}$ L. Schnieder, W. Meier, K. H. Welge, M. N. R. Ashfold, and C. M. Western, "Photodissociation dynamics of $\mathrm{H}_{2} \mathrm{~S}$ at $121.6 \mathrm{~nm}$ and a determination of the potential-energy function of SH( $\left(\mathrm{A}^{2} \Sigma^{+}\right)$," J. Chem. Phys. 92, 7027-7037 (1990).
${ }^{25}$ Y. Dorenkamp, C. Volkmann, V. Roddatis, S. Schneider, A. M. Wodtke, and $\mathrm{O}$. Buenermann, "Inelastic $\mathrm{H}$ atom scattering from ultrathin aluminum oxide films grown by atomic layer deposition on $\mathrm{Pt}(111)$, , J. Phys. Chem. C 122, 10096-10102 (2018).

${ }^{26}$ F. Reinert and G. Nicolay, "Influence of the herringbone reconstruction on the surface electronic structure of Au(111)," Appl. Phys. A 78, 817-821 (2004). 